\title{
Desponte semi-mecanizado como alternativa de otimização da mão-de- obra e redução dos custos operacionais do manejo de poda verde da videira
}

\author{
Semi-mechanized shoot topping as an alternative for workforce optimization and reduction of \\ operational costs of summer pruning management of grapevines
}

\author{
Douglas André Würz ${ }^{1 *}$, Alberto Fontanella Brighenti ${ }^{2}$, Ricardo Allebrandt ${ }^{1}$, Betina Pereira de Bem ${ }^{1}$, \\ Bruno Farias Bonin ${ }^{1}$, José Luiz Marcon Filho ${ }^{3}$, Emilio Brighenti' ${ }^{2}$ Leo Rufato ${ }^{1}$ \\ ${ }^{1}$ Universidade do Estado de Santa Catarina, Lages, SC, Brasil. *Autor para correspondência: douglaswurz@hotmail.com. \\ ${ }^{2}$ Vinícola Legado, Campo Largo, PR, Brasil. \\ ${ }^{3}$ Empresa de Pesquisa Agropecuária e Extensão Rural de Santa Catarina, São Joaquim, SC, Brasil.
}

Submissão: 29/05/2017 | Aceite: 13/06/2018

\begin{abstract}
RESUMO
O objetivo deste trabalho foi verificar a influência de métodos de desponte mecanizado na otimização da mão-de-obra e redução de custos operacionais do desponte da videira em região de elevada altitude de Santa Catarina. O trabalho foi conduzido na Estação Experimental de São Joaquim (Epagri), na safra 2016. O desponte foi realizado durante a fase 75 na escala $\mathrm{BBCH}$. Os tratamentos consistiram em três diferentes métodos de desponte da videira: desponte manual, desponte manual com plataforma acoplada em trator e desponte semi-mecanizado com podador mecânico com auxílio de plataforma acoplada em trator. O delineamento experimental utilizado foi o inteiramente casualizado. Os dados foram submetidos $a$ análise da variância, sendo realizado o teste Tukey $(p \leq 0,05)$. O desponte semi-mecanizado apresentou uma redução de $1.106 \%$ no tempo para realização do desponte em comparação com o método manual. Verificou-se um custo de $\mathrm{R} \$ 289,70 \mathrm{ha}^{-1}$ para o desponte semi-mecanizado, enquanto o desponte manual apresentou um custo de $R \$ 665,00$ ha $^{-1}$, enquanto o desponte manual com plataforma apresentou um custo de $\mathrm{R} \$ 766,40$ ha $^{-1}$. A semi-mecanização do desponte com a utilização de um podador mecânico com plataforma acoplada ao trator reduziu os custos dessa prática de manejo cultural, otimizando o uso de mão-de-obra.
\end{abstract}

PALAVRAS-CHAVE: Vitis vinifera L., dossel vegetativo, mecanização, vitivinicultura.

\begin{abstract}
The objective of this study was to verify the influence of mechanized shoot topping in workforce optimization and reduction of operational costs to perform the grapevine pruning in the highlands of Santa Catarina, Brazil. The experiment was conducted at São Joaquim Experimental Station during the 2016 crop. Shoot topping was performed during phase 75 of the $\mathrm{BBCH}$ scale. The treatments consisted of three different shoot topping methods: manual shoot topping, manual shoot topping using a platform coupled to a tractor and semi-mechanized shoot topping performed with a mechanical pruner using a platform coupled to a tractor. The experimental design was completely randomized. Data were submitted to analysis of variance (ANOVA), and Tukey's test was performed ( $\leq \leq 0.05)$. The semi-mechanized pruning method presented a $1,106 \%$ reduction in time to perform shoot topping in comparison to the manual method. The cost for semi-mechanized topping was $\mathrm{R} \$ 289.70 \mathrm{ha}^{-1}$, manual topping costed $\mathrm{R} \$ 665.00 \mathrm{ha}^{-}$ 1 , and manual topping with the platform costed $R \$ 766.40 \mathrm{ha}^{-1}$. The semi-mechanization of shoot topping with a mechanical pruner using a platform coupled to a tractor reduces the production costs, in addition to optimizing the use of workforce.
\end{abstract}

KEYWORDS: Vitis vinifera L., vegetative canopy, mechanization, viticulture.

A videira caracteriza-se por ser uma espécie exigente em tratos culturais, e para se alcançar condições ótimas no momento da colheita é fundamental que as técnicas de manejo sejam adequadas as características de cada região (MARCON FILHO et al. 2015). Na viticultura das regiões de elevadas altitudes de Santa Catarina, Brasil, encontram-se condições edafoclimáticas de elevada disponibilidade 
hídrica e solos com altos teores de matéria orgânica (>3,5\%), que promovem o excessivo crescimento vegetativo em detrimento do desempenho produtivo das videiras (ZALAMENA et al. 2013, MARCON FILHO et al. 2015). Na videira, assim como na maioria das espécies frutíferas, o balanço entre a carga de frutas (dreno) e a área foliar adequadamente iluminada (fonte) influenciam na quantidade e na qualidade da produção. $O$ equilíbrio entre esses dois parâmetros é determinante para a composição e a maturação das bagas (MARCON FILHO et al. 2015), sendo o desponte, um dos manejos mais comuns para controlar o excessivo crescimento vegetativo e promover o equilíbrio vegeto-produtivo, melhorando a maturação da videira cultivada em regiões de elevada altitude (BRIGHENTI et al. 2010, VANDERLINDE et al. 2017).

De acordo com VIANNA et al. (2016), a região dos vinhos de altitude caracteriza-se por apresentar pequenas áreas de vinhedo, sendo que o município de São Joaquim apresenta o maior número de propriedades, com área média de 7,6 ha/propriedade. Essa pequena área da propriedade pode ser considerada um entrave para investimentos de mecanização nas práticas de manejo dos vinhedos. Desta forma, na maioria das vezes os tratos culturais da videira são realizados de forma manual, o que acarreta em um maior tempo de execução, maior demanda de mão-de-obra e consequentemente elevação dos custos de produção.

Diversos trabalhos relatam que o aumento dos custos de produção, associados a uma escassez de mão-de-obra representam um risco para a vitivinicultura em diversas regiões do mundo, sendo necessário investimentos em mecanização para o manejo e condução dos vinhedos, mantendo a atividade viável economicamente (BATES \& MORRIS 2009, KURTURAL et al. 2012, GAMBELLA \& SARTORI 2014, FIDELIBUS 2014).

Nesse contexto, o objetivo deste trabalho é de verificar a influência de métodos de desponte semimecanizado na otimização da mão-de-obra e redução de custos operacionais para realização da poda verde da videira em regiões de elevada altitude de Santa Catarina.

O trabalho foi conduzido na Estação Experimental de São Joaquim (Epagri/ EESJ), (28¹7'39"S, $49^{\circ} 55^{\prime} 56^{\prime \prime} \mathrm{W}$, altitude: $1.415 \mathrm{~m}$ ), na safra 2016. O clima da região é mesotérmico úmido (Cfb) segundo a classificação de Köppen-Geiger, ou seja, clima temperado constantemente úmido, sem estação seca, com verão fresco (BENEZ 2005). O solo do campo experimental é classificado como Cambissolo Húmico, de acordo com o Sistema Brasileiro de Classificação de Solos (SANTOS et al. 2013). O vinhedo se caracteriza por apresentar espaçamento de 3,0 × 1,5 m, em filas dispostas no sentido $\mathrm{N}-\mathrm{S}$, conduzidas em espaldeira, podadas em cordão esporonado duplo, a 1,2 $\mathrm{m}$ de altura. O desponte foi realizado na segunda quinzena de dezembro, durante o estádio fenológico de grão ervilha, que corresponde a fase 75 na escala $\mathrm{BBCH}$ proposta por LORENZ et al. (1994).

Os tratamentos consistiram em três diferentes métodos de desponte da videira através de diferentes métodos de intervenção: desponte manual (realizado com tesoura Felco 8), desponte manual (realizado com tesoura Felco 8) com auxílio de plataforma acoplada em trator, desponte semi-mecanizado (realizado com podador Stihl HS $82 \mathrm{R}$ ) com auxílio de plataforma acoplada em trator. O desponte consistiu na retirada de $30 \mathrm{~cm}$ do ápice do dossel vegetativo, mantendo uma área foliar de $4,5 \mathrm{~m}^{2}$ por planta, adequada para a maturação da uva nas condições de altitude elevada de Santa Catarina. Foram despontados 0,1 hectare para cada tratamento, ou seja, 222 plantas para cada intervenção de desponte da videira.

Para determinar o tempo necessário para realizar o manejo do desponte em um hectare de vinhedo (horas), foi cronometrado o tempo gasto para despontar uma planta, sendo este valor multiplicado pela densidade de plantas por hectare (2.222 plantas por hectare). O custo para realizar o manejo do desponte da videira ( $\mathrm{R} \$$ por hora) em um hectare de vinhedo foi determinando multiplicando-se o tempo gasto para realização do manejo do desponte (horas por hectare) pelo custo operacional total de realização da intervenção de manejo em uma hora $(R \$)$. O custo operacional total foi determinado pela soma do custo operacional do equipamento, custo operacional do trator, e custo operacional do tratorista e do operador do manejo do desponte. O custo operacional do equipamento (podador Stihl HS 82 R) foi determinado multiplicando-se o consumo de combustível em uma hora de trabalho, pelo preço médio do combustível no município de São Joaquim, Santa Catarina. O custo operacional do trator (Yanmar 1175) foi determinado multiplicando-se o consumo de combustível em uma hora de trabalho, pelo preço médio do combustível no município de São Joaquim, Santa Catarina. O custo operacional do operador do equipamento de desponte em uma hora de trabalho foi determinado pelo salário mínimo regional catarinense, sendo este $R \$ 1.009,00$ para o setor agropecuário, e para operador de trator considerou-se o salário mínimo catarinense para 0 setor agropecuário adicionado $30 \%$ do valor referente a periculosidade, totalizando $R \$ 1.311,70$.

Através de pesquisa em revendedoras de equipamentos agrícolas, foi realizado o custo médio para aquisição dos equipamentos utilizados para a realização do manejo do desponte, para verificar a 
necessidade de investimento inicial do vitivinicultor, bem como determinar o tempo de retornos do investimentos realizados com os equipamentos utilizados no manejo do desponte da videira (Tabela 1).

Tabela 1. Custo de aquisição dos equipamentos em Reais $(R \$)$ para intervenção mecânica do manejo do desponte da videira, e custo operacional em Reais $(R \$)$ dos equipamentos de desponte, trator, operador do equipamento, operador do trator e custo operacional total em Reais ( $R \$$ ) durante a safra 2016.

Table 1. Cost of equipment acquisition in Reais $(R \$)$ for mechanical intervention of grapevine shoot topping management, and operational cost in Reais $(R \$)$ of shoot topping equipment, tractor, equipment operator, tractor operator and total operating cost in Reais $(R \$)$ during the 2016 crop.

\begin{tabular}{llll}
\hline \multirow{2}{*}{ Custo/Modelo } & \multicolumn{3}{c}{ Método de Desponte } \\
\cline { 2 - 4 } & $\begin{array}{l}\text { Tesoura } \\
\text { Manual }\end{array}$ & $\begin{array}{l}\text { Tesoura com } \\
\text { plataforma }\end{array}$ & $\begin{array}{l}\text { Podador com } \\
\text { plataforma }\end{array}$ \\
\cline { 2 - 4 } & Felco 8 & Felco 8 & Podador Stihl HS 82 R \\
\hline Aquisição do Equipamento $(\mathrm{R} \$)$ & 189,05 & 189,05 & $2.199,00$ \\
Operacional Equipamento $\left(\mathrm{R} \$\right.$ hora $\left.^{-1}\right)$ & 0,00 & 0,00 & 0,72 \\
Operacional Trator $\left(\mathrm{R} \$\right.$ hora $\left.^{-1}\right)$ & 0,00 & 10,80 & 10,80 \\
Operacional Operador $\left(\mathrm{R} \$\right.$ hora $\left.^{-1}\right)$ & 4,59 & 10,55 & 10,55 \\
Operacional Total $\left(\mathrm{R} \$\right.$ hora $\left.^{-1}\right)$ & 4,59 & 21,35 & 22,06 \\
\hline
\end{tabular}

O delineamento experimental utilizado foi o inteiramente casualizado, sendo avaliadas 222 plantas por tratamento, correspondente a 0,1 ha de vinhedo. As variáveis foram submetidas à análise de variância (ANOVA) e quando detectadas efeitos de tratamento, procedeu-se o teste de comparação de médias pelo teste Tukey a $5 \%$ de probabilidade de erro.

Observou-se influência do método de desponte no tempo gasto para realizar essa prática de manejo cultural em um hectare de vinhedo (Tabela 2). No método manual do desponte, utilizando-se tesoura de poda, foram necessárias 144,9 horas para realização do desponte de todas as plantas em um hectare. Já com o uso da plataforma associado ao desponte manual com tesoura observou-se uma maior eficiência de trabalho, sendo necessárias 35,9 horas para despontar um hectare de vinhedo. O método de desponte mais eficaz na redução do tempo de desponte foi o semi-mecanizado, com a utilização do podador Stihl HS $82 \mathrm{R}$ associado com a plataforma, onde houve uma redução de $97 \%$ no tempo para realização de desponte em relação ao desponte manual, sendo que um hectare de vinhedo foi despontado em 4,26 horas. Nesse contexto, o uso do podador associado a plataforma reduziu significativamente o tempo gasto para a realização do manejo do desponte, otimizando a mão-de-obra para o vitivinicultor. Em um estudo realizado por FIDELIBUS (2014), na Califórnia observou-se que com a dificuldade de quantidade de mão-de-obra e o elevado custo de produção, a mecanização torna-se uma alternativa viável para a manutenção da atividade agrícola, caso contrário, pode-se verificar reduções das áreas cultivadas.

Tabela 2. Influência do método de desponte no tempo para realizar o manejo do desponte em uma planta (segundos), em um hectare (horas) e o custo em Reais $(R \$)$ para realizar o manejo do desponte em uma planta e em um hectare de vinhedo durante a safra 2016.

Table 2. Influence of shoot topping method on time to perform topping management in one plant (seconds), in one hectare (hours) and cost in Reais ( $R \$$ ) to perform shoot topping management in one plant and in one hectare of vineyard during the 2016 crop.

\begin{tabular}{lrccc}
\hline & \multicolumn{4}{c}{ Método de Desponte } \\
\cline { 2 - 4 } \multicolumn{1}{c}{ Custo/Modelo } & $\begin{array}{r}\text { Tesoura } \\
\text { Manual }\end{array}$ & Tesoura com plataforma & Podador com plataforma & \\
& Felco 8 & Felco 8 & Podador Stihl HS 82 R & \\
\cline { 2 - 4 } & $58,3 \mathrm{~b}$ & $46,9 \mathrm{~b}$ & $4,26 \mathrm{a}$ & 30,1 \\
Tempo desponte/planta $(\mathrm{seg})$ & $144,9 \mathrm{c}$ & $35,9 \mathrm{~b}$ & $13,1 \mathrm{a}$ & 30,4 \\
Tempo desponte/hectare $(\mathrm{h})$ & $0,30 \mathrm{~b}$ & $0,34 \mathrm{~b}$ & $0,13 \mathrm{a}$ & 29,5 \\
Custo desponte $\left(\mathrm{R} \$\right.$ planta $\left.^{-1}\right)$ & $665,0 \mathrm{~b}$ & $766,4 \mathrm{~b}$ & $289,7 \mathrm{a}$ & 29,8 \\
Custo desponte $\left(\mathrm{R} \$\right.$ hectare $\left.^{-1}\right)$ & & &
\end{tabular}

Médias seguidas da mesma letra, na linha, não diferem entre si pelo teste Tukey a $5 \%$ de probabilidade de erro. 
Em relação ao custo operacional total (custo operacional do equipamento + custo operacional do trator + custo operacional do operador do trator e equipamento), verificou-se que o podador Stihl HS $82 \mathrm{R}$ apresentou um custo de $R \$ 22,06$ por hora trabalhada, enquanto o custo do desponte manual associado com a plataforma apresentou um custo de $R \$ 21,36$ por hora trabalhada e o custo operacional do desponte manual foi de $R \$ 4,59$ por hora trabalhada. Ressalta-se que apesar do desponte realizado com o podador associado a plataforma possuir um custo operacional mais elevado, esse método apresentou o menor custo de manejo do desponte por hectare de vinhedo, visto que há uma drástica redução no tempo necessário para realização da atividade. De acordo com BARTSCH (2010), as intervenções mecânicas são necessárias, principalmente para a redução do custo de produção.

Considerando o custo total para realização do manejo do desponte em um hectare de vinhedo, verifica-se um valor de $R \$ 289,70$ ha $^{-1}$ utilizando-se o manejo semi-mecanizado, $R \$ 766,40$ ha $^{-1}$ para desponte manual associado com a plataforma e $R \$ 665,00 \mathrm{ha}^{-1}$ para desponte apenas manual.

O custo de aquisição do podador Stihl HS 82 R é de $R \$ 2.199,00$, enquanto o custo de aquisição de uma tesoura Felco 8 é de $R \$ 189,05$. No entanto, através das relações de área de vinhedo e custo para realização do manejo do desponte da videira, verificou-se que em um vinhedo com área de 4,2 hectares e 5,3 hectares, a redução do custo para realizar o manejo do desponte com o podador HS 82 R são suficientes para compensar o custo de aquisição do equipamento em relação ao desponte manual associado a plataforma e ao desponte manual sem a plataforma, respectivamente. Em trabalho realizado por BATES \& MORRIS (2009), observou-se que apesar de haver um alto custo para converter um vinhedo à um manejo mecanizado, o investimento realizado acaba retornando pela redução do tempo para realizar o manejo da videira.

Portanto, a semi-mecanização do manejo do desponte da videira com a utilização de um podador mecânico associado a plataforma acoplada ao trator, reduz os custos dessa importante prática cultural, otimizando o uso de mão-de-obra, o que reflete na realização do manejo do desponte no momento fenológico adequado, além de reduzir os custos de produção vitícola.

\section{REFERÊNCIAS}

BARTSCH T. 2010. The cost of cane pruning in a VSP canopy. The Australian and New Zealand Grapegrower and Winemaker 558: 34-36.

BATES T \& MORRIS J. 2009. Mechanical cane pruning and crop adjustment decreases labor costs and maintains fruit quality in New York 'Concord' grape production. HortTechnology 19: 247-253.

BENEZ MC. 2005. Dados e informações biofísicas da Unidade de Planejamento Regional Planalto Sul Catarinense UPR 3. In: DUFLOTH J.H et al. Estudos básicos regionais de Santa Catarina. Florianópolis: Epagri. 76p.

BRIGHENTI AF et al. 2010. Desponte dos Ramos da videira e seu efeito na qualidade dos frutos de 'Merlot' sobre os porta-enxertos 'Paulsen 1103' e 'Courdec 3309'. Revista Brasileira de Fruticultura 32: 19-26.

FIDELIBUS MW. 2014. Grapevine cultivars, trellis systems, and mechanization of the California raisin industry. HortTechnology 24: 285-289.

GAMBELLA F \& SARTORI L. 2014. Comparison of mechanical and manual cane pruning operations on three varieties of grape (Cabernet Sauvignon, Merlot and Prosecco) in Italy. Transactions of the ASABE 57: 701-707.

KURTURAL SK et al. 2012. Mechanical canopy management reduces labor costs and maintains fruit composition in 'Cabernet Sauvignon' grape production. HortTechnology 22: 509-516.

LORENZ DH. 1994. Phänologische Entwicklungsstadien der Weinrebe (Vitis vinifera L. ssp. vinifera). Codierung und Beschreibung nach der erweiterten BBCH-Skala. Viticulture and Enology Science 49: 66-70.

MARCON FILHO JL et al. 2015. Raleio de cachos sobre o potencial enológico da uva 'Cabernet Franc' em duas safras. Ciência Rural 45: 2150-2156.

SANTOS HG et al. 2013. Sistema brasileiro de classificação de solos. 3.ed. Brasília: Embrapa. 342p.

VANDERLINDE G et al. 2017. Influence of the timing of shoot topping on yield and grape quality in 'Cabernet Sauvignon' and 'Merlot' cultivars. Acta Horticulturae 1157: 407-412.

VIANNA LF et al. 2016. Caracterização agronômica e edafoclimáticas dos vinhedos de elevada altitude. Revista de Ciências Agroveterinárias 15: 215-226.

ZALAMENA J et al. 2013. Estado nutricional, vigor e produção em videiras cultivadas com plantas de cobertura. Revista Brasileira de Fruticultura 35: 1190-1200. 